\title{
A teoria da agência no setor da saúde: o caso do relacionamento da Agência Nacional de Saúde Suplementar com as operadoras de planos de assistência supletiva no Brasil*
}

\author{
Fábio Ricardo Loureiro Sato**
}

\begin{abstract}
SumÁrio: 1. Introdução; 2. A teoria da agência; 3. A regulamentação do sistema de saúde suplementar; 4 . Os atuais conflitos do setor sob a ótica da teoria da agência; 5 . Seleção adversa e redução do risco; 6 . O risco moral no setor de assistência supletiva; 7. Conclusão.
\end{abstract}

Sum MARY: 1. Introduction; 2. Agency theory; 3. Regulation of the supplementary healthcare system; 4. Current conflicts in the sector from the agency theory standpoint; 5. Adverse selection and risk reduction; 6. Moral risk in the supplementary healthcare sector; 7. Conclusion.

Palavras-CHAVE: teoria da agência; setor de assistência supletiva à saúde.

KEY WORD S: agency theory; supplementary healthcare sector.

O relacionamento entre os atores do sistema supletivo de assistência à saúde no Brasil é caracterizado por uma complexa trama de conflitos de interesse e de poder, que podem ser analisados à luz da teoria da agência. Ela emprega elementos da ciência econômica, principalmente das teorias do consumidor e do funcionamento de mercado, conjuntamente com idéias derivadas dos estudos organizacionais e comportamentais, faz uma divisão entre os agentes econômicos em principais e agentes. Analisando o mercado de assistência supletiva, pode-se constatar que ele possui inúmeros atores, que exercem ao mesmo tempo papéis de agentes e principais. É justamente dessa dualidade de papéis que surgem grande parte dos conflitos, e, através de diversos mecanismos de regulação, tenta-se reduzir os custos de agência derivados dos conflitos de interesse que são a causa principal dos problemas de agência dentro desse setor.

\footnotetext{
* Artigo recebido em jul. 2004 e aceito em dez. 2005.

** Mestre em administração hospitalar e de sistemas de saúde pela Eaesp/FGV. Endereço: Rua Santa Catarina, 418, ap. 101 — Tatuapé - CEP 03086-025, São Paulo, Brasil. E-mail: fsato@gvmail.br.
} 
Agency theory in the healthcare sector: the relationship between the National Supplementary Healthcare Agency and the supplementary healthcare operators in Brazil

The relationship between the actors of the supplementary healthcare system in Brazil is characterized by complex conflicts of interests and power struggles, which can be analyzed by the agency theory. This theory - combining elements of economic sciences, mainly consumer and market theories, with ideas derived from organizational and behavioral studies - divides the economic agents in principals and agents. The analysis of the supplementary healthcare market reveals its many actors play simultaneously the roles of agent and principal. This duality of roles is the root of most conflicts, originating several regulation mechanisms which try to reduce agency costs derived from conflicts of interests that are the main cause of this sector's agency issues.

\section{Introdução}

Nos sistemas nacionais de saúde, a oferta, o financiamento, a operação e a regulação dos serviços são, em geral, prerrogativas da associação entre as iniciativas pública e privada. E as características e organização dessas ações variam, interna e externamente, nos diferentes países, de acordo com o contexto histórico.

Após a II Guerra Mundial, consolidou-se a tendência da instituição de sistemas integrados públicos. Neles, cabiam ao Estado o financiamento e a provisão de serviços. Era a época do conhecido Estado social keynesiano.

Com o passar dos anos, esse modelo de welfare state entrou num período de crise e foi sendo substituído por um modelo neoliberal. Nesse contexto, constatam-se evidências de mudanças na participação pública e privada.

A atual fase dos sistemas de saúde caracteriza-se pela introdução de mecanismos de mercado na provisão dos serviços, pelas proposições de renúncia do Estado à responsabilidade por essas funções e pelo fortalecimento da participação da oferta e das despesas privadas em saúde, ainda que a carta magna do país afirme a universalidade, a integralidade e a eqüidade do sistema, o que institui a dualidade público-privado dentro do sistema de saúde brasileiro.

Com o crescimento do setor privado na assistência à saúde, com destaque especial ao setor supletivo, o Estado que acabou por se afastar em parte da prestação dos serviços passou a ganhar importância na regulamentação do setor privado.

É justamente nesse contexto de regulamentação que nasceu a Agência Nacional de Saúde Suplementar (ANS). A ANS tem como função fiscalizar e regulamentar os contratos entre os usuários e as operadoras de saúde. Entretanto, o impacto não se restringe a esses dois atores. Todos os prestadores, 
como hospitais, laboratórios, profissionais, entre outros, são influenciados pelas medidas tomadas pela ANS.

É justamente nesse contexto de regulação que surgem os chamados conflitos de interesse entre os atores desse setor: a ANS procurando defender os usuários dos planos de saúde, que possuem demandas divergentes das operadoras que, por sua vez, têm poder de barganha sobre fornecedores, que acabam sendo pressionados por outros fatores conjunturais, e podem contestar muitas das decisões das empresas de planos de saúde.

Parte desses conflitos pode ser avaliada à luz da teoria da agência, que procura analisar as relações e conflitos existentes entre os diversos atores de uma determinada rede de relacionamento.

Assim, este artigo faz uma análise do relacionamento entre os atores do mercado de assistência supletiva à saúde tendo como base o referencial teórico da teoria da agência.

\section{A teoria da agência}

De acordo com Jensen e Meckling (1976), a teoria da agência trata do relacionamento entre agentes nas trocas econômicas, onde um ator (o principal) tem poder sobre o comportamento de um outro ator (o agente) em seu favor, e o bem-estar do principal sofre influência das decisões do agente.

Figura 1

\section{A relação principal-agente}

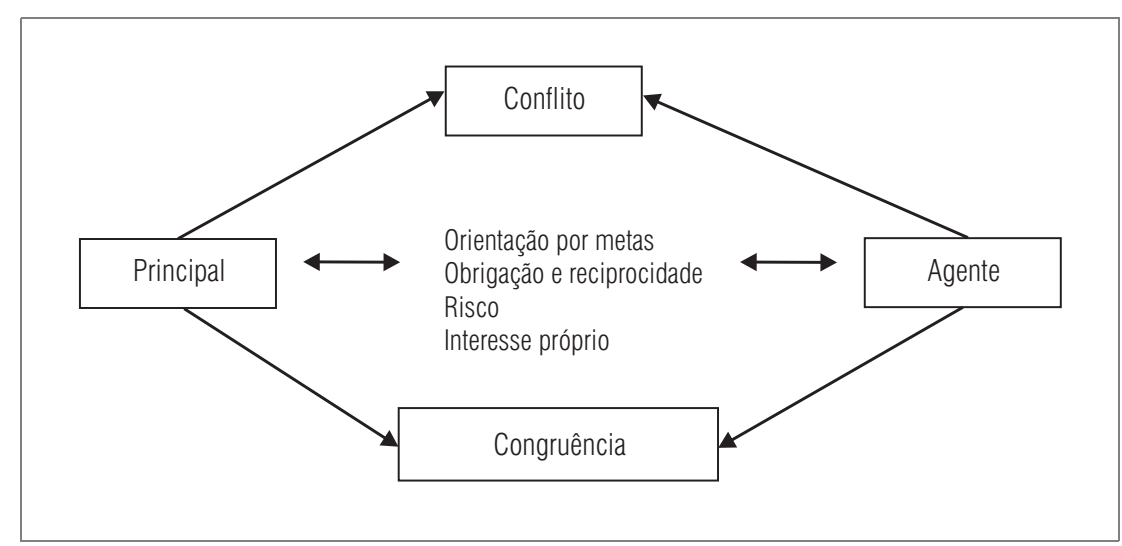

Rap Rio de Janeiro 41(1):49-62, Jan./Fev. 2007 
Dessa forma, o bem-estar do principal não pode ser maximizado porque o principal e o agente possuem diferentes objetivos e predisposição ao risco (Wright et al., 1996). O principal pode ser considerado neutro quanto ao risco, pois pode realizar escolhas sobre uma ampla gama de participantes (Wiseman e Gomez-Mejia, 1998). Em contraste, o agente precisa agir de forma adversa ao risco, já que está ligado a um único principal (Willianson, 1963). Em essência, os agentes são em princípio adversos ao risco como forma de preservarem seus bens. Portanto, a teoria da agência tem como foco o estudo das formas de minimização dos custos relacionados com a relação de agência.

O problema de agência, segundo Hatch (1997), envolve o risco do agente agir de acordo com seus interesses em vez dos do principal. Para evitar os problemas de divergência de interesses, são realizados contratos, cujo objetivo é alinhar os interesses dos agentes com os de seus principais.

Para Eisenhardt (1985), a habilidade dos principais em saberem se os agentes estão ou não agindo de acordo com seus interesses, depende das informações disponíveis aos principais. Essas informações podem ser obtidas diretamente pelo monitoramento direto das ações dos agentes, ou, indiretamente, pelo acompanhamento dos resultados produzidos pelos agentes. Entretanto, como os resultados não dependem apenas dos agentes, eles nesses casos passam a assumir certa parcela do risco.

A fim de proteger os interesses dos principais, é necessária a instituição de mecanismos que reduzam a possibilidade de os agentes agirem em desacordo. Nessa tarefa, custos são incorridos e denominados, segundo Clegg, Hardy e Nordy (1996), custos de agência. Os custos totais de agência são o montante gasto no monitoramento pelos principais, os custos da dependência dos agentes e a perda residual do principal.

Assumindo que os custos de agência existem, tanto os principais quanto os agentes atuam de forma a minimizar esse custo e, portanto, ficar numa situação de equilíbrio entre ambas as partes.

Arrow (1985) identificou duas fontes principais de problemas de agência: o moral hazard, ou risco moral, que está relacionado com acobertar ações, e a seleção adversa, relacionada a acobertar informações. O risco moral envolve situações onde as ações dos agentes não são de conhecimento do principal ou são muito onerosas para serem observadas. Já a seleção adversa representa o fato de os agentes possuírem informações desconhecidas por parte do principal ou cujos custos de obtenção são elevados.

Os problemas de agência são resolvidos normalmente de duas maneiras: monitoramento e penalização. Para Clegg, Hardy e Nord (1996), o monitoramento envolve a observação da performance dos agentes, e a penalização é a punição por um comportamento não desejado dos agentes. 


\section{A regulamentação do sistema de saúde suplementar}

O Decreto-Lei $n^{0} 73 / 66$, referente à conformação do sistema nacional de seguros, foi o primeiro instrumento legal sobre a comercialização de planos e seguros de saúde. Em função de divergências entre entidades representantes dos médicos, empresas de medicina de grupo que necessitavam de proteção para se desenvolver, essa legislação não abrangeu as operadoras "nãolucrativas". Desde então, empresas de medicina de grupo "não-lucrativas" e cooperativas médicas operaram no mercado sem nenhuma regulamentação específica (Bahia, 2001).

A expansão do setor supletivo que se deu na década de 1980 foi acompanhada pelo crescimento no número de queixas de interrupção de atendimento, aumento de preços e negativas de cobertura para determinados procedimentos. O Código de Defesa do Consumidor permitiu a ação da Justiça no sentido da concessão de liminares favoráveis ao atendimento de usuários do setor supletivo. Junto a isso, entidades de defesa do consumidor e entidades médicas se aliaram em torno de interesses comuns como a liberdade de escolha, e a mídia passou a divulgar constantemente matérias de forma a divulgar casos onde houve restrições de atendimentos por parte das operadoras de saúde.

Em 1993, começaram a tramitar no Senado e na Câmara Federal projetos pontuais de regulamentação do setor supletivo de assistência à saúde. Esses projetos tinham dois objetivos centrais: ampliação da cobertura (procedimentos e faixas etárias) e ressarcimento monetário do atendimento de clientes em instituições públicas. Em 1997, foi constituída uma comissão especial para tratar da regulamentação e, em 1998, a Câmara aprovou a Lei nº 9.656, que obrigou a, entre outras coisas (Brasil, 1998):

v atendimento para todas as doenças relacionadas no Código Internacional de Doenças;

\ proibição de negação de cobertura em função de doenças ou lesões preexistentes;

v ressarcimento pelas operadoras dos atendimentos prestados a seus clientes na rede vinculada ao SUS.

O arcabouço legal previsto pela Lei $\mathrm{n}^{\mathrm{o}}$ 9.656/98, alicerçado na ampliação e padronização das coberturas, estimula mudanças nas relações entre provedores de serviços e operadoras de planos de saúde, na medida da inten- 
sificação da indiferenciação de produtos prevista pela lei. A legislação supõe que a competição entre as operadoras será baseada apenas nos preços e nos modelos de regulação do acesso de clientes e médicos à utilização de procedimento e não em padrões diferenciados de cobertura.

Com a finalidade de fiscalizar o cumprimento da legislação pertinente ao setor, foi criada, em 30 de dezembro de 1999, a Agência Nacional de Saúde Suplementar (ANS), pela Medida Provisória no 2.012-2. De acordo com Nunes (1999), esta agência regulatória deveria ter como características: autonomia de decisão; ampla divulgação de decisões e procedimentos; celeridade nas relações com os consumidores e agentes econômicos; inclusão dos interessados em audiências públicas e limitação da ação estatal na provisão de serviços públicos.

De acordo com Moreira (2004), a criação de uma entidade reguladora da saúde pode ser traduzida efetivamente na criação de um mercado de cuidados de saúde, em que os atores principais deixam de ser estabelecimentos públicos sem a lógica empresarial, de um lado, e pacientes de serviços públicos gratuitos de outro, para passarem a ser entidades empresariais, sujeitas a uma lógica de mercado, sendo remuneradas pelos cuidados efetivamente prestados, e consumidores de cuidados de saúde que são remunerados aos prestadores, embora não necessariamente pelos próprios consumidores. É interessante ainda ressaltar que as agências incentivam muito a competitividade nos setores regulados e a desconcentração de capitais. Portanto, estão de acordo com a idéia de que os mercados promovem maiores ganhos sociais à medida que seus participantes são mais numerosos.

Entre as funções a serem exercidas pela ANS, podemos citar:

V a combinação de estratégias coercitivas e cooperativas para as empresas que atuam no setor econômico, sejam orientadas ou não para o mercado;

V a aceitação de que a competição pode gerar equilíbrio nas decisões econômicas e favorecer o direito de consumidores, e que as condutas responsáveis das empresas podem trazer benefício público (externalidade positiva);

v o desenvolvimento de estratégias cooperativas entre as estruturas institucionais do SUS (Sistema Único de Saúde) e os segmentos da saúde supletiva, diante da atenção de alto custo e de longa duração;

- o acompanhamento e a avaliação das orientações redistributivas, como o ressarcimento ao SUS. 


\section{Os atuais conflitos do setor sob a ótica da teoria da agência}

\section{Ótimo de Pareto e seleção adversa}

A teoria da agência argumenta que a informação é naturalmente assimétrica entre agentes e principal e que, quando os conflitos de interesse existem, agentes e principais podem fazer uso de informações exclusivas e do poder de coerção para influenciar contratos que estejam demasiadamente favoráveis a uma determinada parte.

Para se atingir um ponto ótimo de um contrato com relação aos custos de agência, é necessário balancear os trade-offs de maximização de benefícios, onde não é possível melhorar os resultados de uma das partes sem prejudicar as demais. Nesse ponto, atingir-se-ia o conhecido ótimo de Pareto (1971).

No setor de assistência supletiva, o ótimo de Pareto seria atingido no equilíbrio entre as maximizações do interesse da ANS - representante legal dos usuários - e das operadoras de planos de saúde. A maximização para a ANS seria o atendimento da demanda por cuidados de saúde dos beneficiários dos planos de saúde, nesse caso, pensando demanda por cuidados de saúde como variável proxi a demanda por saúde, conforme proposta de Grossman (1972).

Já as operadoras de planos de saúde podem ser divididas segundo a sua modalidade de funcionamento para analisar a maximização da sua função utilidade. Para as autogestões, a maximização seria obtida pelo atendimento das demandas de seus beneficiários, tendo como princípio a não-lucratividade. Entretanto, é preciso sempre considerar a existência de um fator de restrição orçamentária.

As demais formas de modalidade de operação, como são os casos das medicinas de grupo, seguradoras e cooperativas, têm como objetivo claro a maximização da sua lucratividade.

Dessa forma, surge nesse ponto o primeiro grande conflito de interesse entre os participantes desse mercado: a ANS, procurando defender os usuários de planos de saúde diante dos planos de saúde, que são certamente a parte mais fraca na relação com as operadoras e que, portanto, estão numa posição de agentes e as operadoras na posição de principal. Com a criação da ANS, uma nova relação agente-principal surge nesse mercado, onde a agência regulatória assume o papel de principal e as operadoras de saúde, o de agentes.

O grande problema que existe nesse mercado é que o ótimo de Pareto é muito difícil de ser atingido. A demanda por saúde segue um padrão com pequena elasticidade de demanda. Conforme afirma Roemer (1993), autor da lei que possui seu epônimo, a oferta de serviços de saúde consegue por si só gerar a sua própria demanda. 
Para piorar essa situação, a inflação dos bens e cuidados de saúde é muito mais elevada do que a média de mercado, conforme mostra Rochaix (1990). Uma possível explicação para esse aumento progressivo da inflação da saúde está na Lei de Baumol (Baumol, 1967), que afirma que ocupações como as da saúde são inerentemente inflacionárias devido ao fato de serem intensivas em mão-de-obra. Para a sociedade, a fonte de riqueza econômica ao longo do tempo é a substituição do trabalho pelo capital, sendo essa a própria definição de produtividade. Entretanto, pessoas qualificadas em ocupações em que o capital não substitui o trabalho esperam ao longo do tempo que sua renda aumente no mínimo em proporção ao aumento médio de renda da sociedade. Portanto, temos uma grande pressão pelo aumento dos custos das operadoras de saúde, que, por sua vez, estabelecem mecanismos de forma a reverterem essa situação e manter o equilíbrio econômico-financeiro dessa relação.

Para isso, as operadoras precisariam estabelecer mecanismos de controle de demanda (como a criação de co-pagamentos ou franquias), a elevação dos preços dos planos de saúde para os beneficiários ou a diminuição dos pagamentos por procedimentos realizados na rede credenciada. Essas medidas até seriam viáveis, já que as operadoras exercem um relevante poder de agência sobre esses atores, porém, essas mesmas operadoras estão numa situação de agentes junto a outras estâncias, como a própria ANS e outras entidades representativas de consumidores, profissionais e instituições de saúde, que contestam tais atitudes se forem praticadas.

As relações de poder na área da assistência supletiva são muito complicadas, o que pode ser constatado por uma breve revisão de alguns fatos que ocorreram no setor. Como exemplo de poder dos beneficiários foi a própria criação da ANS, também muito impulsionada por entidades como os Procons (Procuradoria do Consumidor) de diversos estados, que já há algum tempo vinham constatando a necessidade de uma legislação específica que regulamentasse o setor.

Já as operadoras, principalmente aquelas de maior porte, possuem grande poder de barganha sobre os beneficiários, principalmente no estabelecimento de barreiras para restrição de acesso e redução de custo. Apesar de não estarem tão difundidas como nos casos das Health Maintenance Organizations (HMOs) norte-americanas, atualmente no Brasil já se começam a observar algumas práticas de managed care, e para as medicinas de grupo menores, já existem casos de empresas operando no formato de (PPOs).

Entretanto, controlando essas operadoras do setor supletivo, temos a ANS, que atua para fiscalizar o cumprimento dos contratos de cobertura de 
custos de cuidados em saúde, que por sua vez devem estar de acordo com a Lei $\mathrm{n}^{\mathrm{O}}$ 9.656/98.

Seria interessante também ressaltar outros grupos de influência, como as entidades representantes das operadoras (Abramge, Fenaseg, Unidas, Unimed Brasil) e dos prestadores (FBH, Anahp, CMB, AMB).

Atualmente outro importante ator nesse mercado é o Poder Judiciário. Muitos usuários de planos de saúde - cujas operadoras se negam a prestar determinado tipo de atendimento/procedimento pelos mais diversos motivos, desde questionando a real necessidade e eficácia dos mesmos, até a questão da preexistência - muitas vezes recorrem à Justiça a fim de garantir seu atendimento.

Infelizmente o Poder Judiciário brasileiro ainda possui uma visão muito paternalista em relação aos processos envolvendo a assistência à saúde, e acaba na grande maioria dos casos dando ganho de causa aos usuários, sem análises mais aprofundadas quanto ao mérito da questão, gerando custos ao agente nessa relação que são as operadoras de planos de saúde.

\section{Seleção adversa e redução do risco}

Como afirma Eisenhardt (1989), a seleção adversa é basicamente um problema de assimetria de informação, o que caracteriza as relações entre os atores da saúde (Arrow, 1963). A seleção adversa é tipicamente manifestada em diferentes divisões de risco. Segundo a teoria da agência, os agentes são mais adversos ao risco, justamente por possuírem menor poder de negociação que seus principais.

De acordo com Shapiro e Mulligan (1996), as instituições que operam no setor de saúde estão expostas aos seguintes riscos:

- risco da operação clínica — risco na variação dos custos incorridos na prestação dos serviços clínicos;

- risco de eventos - associado à flutuação na demanda por cuidados de saúde na população de beneficiários;

- risco de precificação - inerente à precificação diante da imprevisibilidade de despesas devido ao risco de eventos;

- risco financeiro - são os riscos básicos a que todas as companhias estão expostas: de capital, insolvência, fluxo de caixa e risco regulatório. 
Diante da existência desses riscos, a questão que se coloca dentro da teoria da agência é a de como fazer o seu compartilhamento. Antes da criação da ANS, os usuários, no papel de agentes, arcavam com uma parcela significativa dos riscos: existiam limites para dias de internação, alguns procedimentos de maior complexidade eram excluídos da cobertura, doenças preexistentes não tinham cobertura, assim como outras como a Aids. Com a instituição da ANS, grande parte dessas assimetrias se reduziu, e as operadoras de planos de saúde são obrigadas a fornecerem a seus beneficiários no mínimo um plano dito referência, com as características já anteriormente citadas que foram incluídas nessa nova legislação.

Além de regulamentar toda a questão de cobertura de custos de procedimentos, a ANS também ficou responsável por estabelecer os reajustes máximos anuais que as empresas poderiam repassar aos seus beneficiários, em função da inflação do período. É justamente nesse ponto que surgem as maiores divergências. Ao mesmo tempo que se aumentou compulsoriamente a oferta de um grande número de procedimentos por parte da ANS, ela atuou de forma a restringir possíveis variações nos preços dos planos de saúde, levando a uma situação de pressão sobre as operadoras, sendo que muitas delas, principalmente as menores e que não tinham boa gestão, acabaram por falir.

Figura 2

Impacto da ANS sobre a curva de demanda

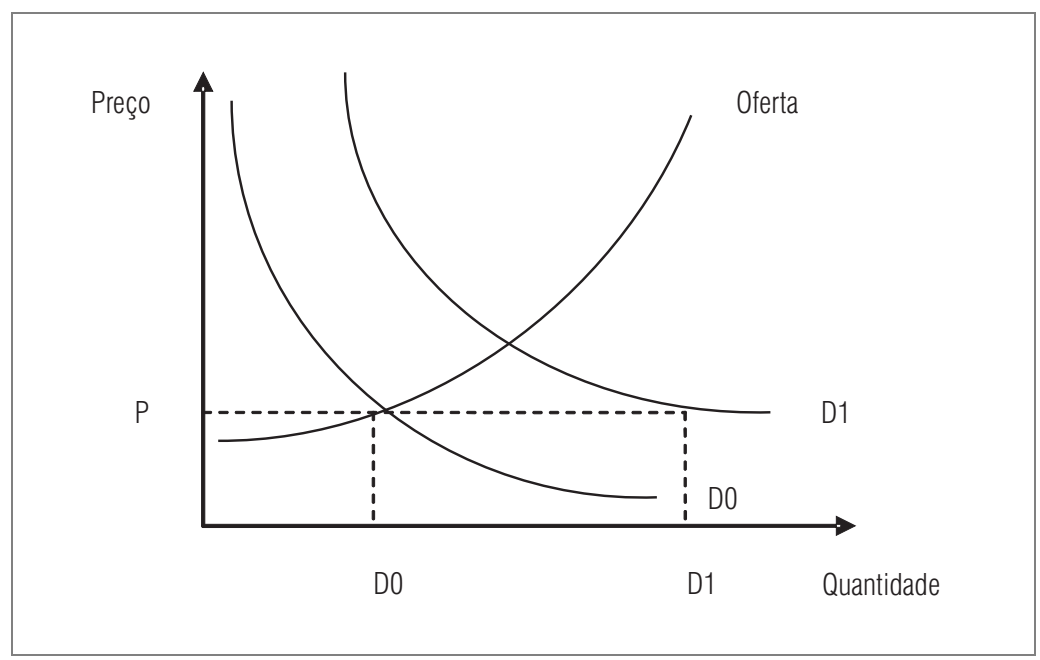

RaP Rio de Janeiro 41(1):49-62, Jan./Fev. 2007 
Além da crítica no que diz respeito ao controle dos preços por parte da ANS, criticam-se também os reajustes permitidos, que invariavelmente ficam abaixo da inflação do setor no período. Nesse caso, fica claro a demonstração do poder de agência por parte da ANS e os custos de agência conforme descrição de Clegg, Hardy e Nord (1996), sendo que as operadoras acabam por arcar com grande parcela deles.

De acordo com Eisenhardt (1989), uma das formas de se reduzirem as assimetrias de informação por parte dos agentes é com o ganho em experiência. Dessa forma, os ganhos de escala também são válidos nessa questão. Assim, operadoras de grande porte e com vasta experiência de mercado tendem a possuir cada vez mais uma posição de oligopólio dentro do mercado, criando barreiras à entrada dos possíveis novos participantes.

\section{O risco moral no setor de assistência supletiva}

O risco moral tem maior importância no relacionamento usuários e operadoras de saúde. De acordo com Pores (1995), o risco moral é facilmente identificado quando comparamos a utilização dos serviços de saúde por duas populações distintas e de perfil social e epidemiológico semelhante, onde os usuários com cobertura de plano de saúde têm tendência a utilizar muito mais o sistema que os sem cobertura.

Em parte, isso está relacionado com a diferenciação entre os conceitos de demanda e necessidade. Para Jeffers, Bognanno e Bartlett (1971), o conceito de necessidade é uma definição exógena, ou seja, a quantidade de serviços de saúde que a opinião médica acredita deva ser consumida em um determinado período de tempo para que as pessoas possam permanecer ou ficar tão saudáveis quanto seja possível segundo o conhecimento científico existente. Já a demanda está centrada sobre a liberdade e a autonomia de escolha do consumidor segundo sua própria estrutura de preferências. Portanto, nesse gap existente entre os conceitos de demanda e necessidade é que se encontra grande parte do conceito econômico que chamamos de indução da demanda. Diversas podem ser as origens da indução da demanda: pressão social, mídia e inclusive os próprios médicos, conforme apontaram Rochaix (1987) e Campos (1983).

Para evitar-se a indução da demanda por serviços de saúde cuja necessidade é no mínimo questionável, é necessária a criação de barreiras por parte do principal para evitar que os agentes atuem em desacordo com seus interesses. Isso já é bastante comum em países onde o setor supletivo já opera há algum tempo, com a adição de fatores de moderação aos contratos de planos de saúde. 
Um desses mecanismos para controle da demanda por serviços de saúde é a implantação do co-pagamento. No co-pagamento, o beneficiário fica responsável pelo pagamento de parte do custo total do prestador, ou seja, ocorre um compartilhamento do risco, ou mais precisamente, da incidência de um determinado sinistro com a saúde do beneficiário. Também pode ser implantada a franquia, principalmente para procedimentos de grande risco, como para internações e cirurgias, onde é descontada do beneficiário uma quantia fixa por evento.

\section{Conclusão}

Como pode ser observado pelas informações anteriormente descritas, o setor de assistência supletiva à saúde no Brasil é muito complexo, com inúmeros atores e motivações que em grande parte dos casos são divergentes entre si. Derivado dessas constatações é possível encontrar inúmeros conflitos de interesse no setor, como são os casos dos beneficiários e as operadoras de planos de saúde, onde cada um deles age de forma a maximizar seus benefícios. Como são divergentes, começa então a surgir o problema de agência, onde os atores que estão no papel de principal em determinado relacionamento (por exemplo, a ANS ou as operadoras de saúde) passam com vários mecanismos de coerção, como o monitoramento e a punição, a controlar o comportamento de seus agentes (respectivamente para o exemplo anterior, operadoras e beneficiários de planos de saúde). É justamente desse controle que incorrem os chamados custos de agência.

Esses custos de agência possuem um custo de oportunidade muito grande, principalmente em se pensando num setor como é o da saúde, com enorme demanda reprimida devido à escassez da oferta derivada de fatores de restrição orçamentária tanto do setor público, quanto do setor privado.

Sendo assim, faz-se necessária a criação de mecanismos que reduzam esses custos de agência e facilitem a obtenção da maximização dos benefícios por todos os atores desse mercado. Baseado nessa afirmação pode-se dizer que a criação da ANS representou um papel importante no sentido de se reduzirem as assimetrias que existiam nesse mercado anteriormente, onde as operadoras tinham grande supremacia sobre seus beneficiários.

Entretanto, é importante ressaltar que o surgimento da ANS não pode representar a criação de novos entraves que tenham muitos impactos negativos no setor, cuja análise custo-benefício (ACB) venha a demonstrar que a instituição dessa agência não conseguiu trazer os retornos positivos esperados pela sociedade. 
Dessa forma, é importante conhecermos e analisarmos como são os relacionamentos do setor, objetivando a harmonização das relações entre os atores, de modo a amenizar os problemas de agências e os custos advindos dos mesmos.

\section{Referências bibliográficas}

ARROW, K. J. Uncertainty and the welfare economics of medical care. American Economic Review, n. 53, 1963.

Principal and agents: the structure of American business. Boston: Harvard Business School Press, 1985.

BAHIA, L. O mercado de planos e seguros de saúde no Brasil: tendências pós-regulamentação. In: NEGRI, B.; DIGIOVANNI, G. Brasil — radiografia da saúde. Campinas: Instituto de Economia da Unicamp, 2001.

BAUMOL, W. J. Macroeconomics of unbalanced growth: the anatomy of urban crisis. American Economy Review, v. 57, n. 3, p. 415-426, Jun. 1967.

BRASIL. Lei n. 9.656, de 3 de junho de 1998. Dispõe sobre os planos e seguros privados de assistência à saúde. Diário Oficial da União, Brasília, 1998.

CAMPOS, A. C. O comportamento do médico como agente econômico. In: CAMPOS, A. C. Saúde, o custo de um valor sem preço. Lisboa: Livros Técnicos e Científicos, 1983.

CLEGG, S. R.; HARDY, C.; NORD, W. R. Handbook of organization studies. London: Sage Publications, 1996.

EISENHARDT, K. M. Control: organizational and economic approaches. Management Science, v. 31, n. 2, p. 134-149, 1985.

Agency theory: an assessment and review. Academy of Management Review, v. 14, n. 1, p. 57-74, 1989.

GROSSMAN, M. On the concept of health capital and the demand for health. Journal of Political Economy, v. 80, n. 2, p. 223-255, 1972.

HATCH, M. J. Organization theory: modern symbolic and postmodern perspectives. New York: Oxford University Press, 1997.

JEFFERS, J. R.; BOGNANNO, M. F.; BARTLETT, J. C. On the demand versus need for medical services and the concept of shortage. American Journal of Public Health, v. 61, n. 1, p. 4663, 1971. 
JENSEN, M.; MECKLING, W. Theory of the firm: managerial behavior, agency costs and capital structure. Journal of Financial Economics, v. 3, p. 305-360, 1976.

MOREIRA, V. A nova entidade reguladora da saúde em Portugal. Revista de Direito Público da Economia, v. 2, n. 5, p. 103-159, jan./mar. 2004.

NUNES, E. Reforma administrativa, reforma reguladora: a nova face da relação Estado-economia no Brasil. 1999. ms.

PARETO, V. Manual of political economy. New York: Augustus Kelley, 1971.

PORES, J. H. Managing risk to reduce costs. Health Financial Management, v. 49, p. 52-56, Oct. 1995.

ROCHAIX, L. O poder discricionário do médico: mito ou realidade. In: CAMPOS, A. C.; PEREIRA, J. Sociedade, saúde e economia. Lisboa: Escola Nacional de Saúde Pública, 1987.

Oferta de cuidados: regulação ou desregulação. Lisboa: Associação Portuguesa de Economia da Saúde, 1990.

ROEMER, M. I. National health systems of the world. New York: Oxford University Press, 1993.

SHAPIRO, M.; MULLIGAN, D. Managing risk in health care. The McKinsey Quarterly, n. 3, p. 94-105, 1996.

WILLIANSON, O. E. Managerial discretion and business behavior. American Economy Review, v. 53, p. 1032-1047, 1963.

WISEMAN, R. M.; GOMEZ-MEJIA, L. R. A behavioral agency model of managerial risk taking. Academy Management Review, v. 23, n. 1, p. 133-153, 1998.

WRIGHT, P. et al. The impact of the corporate insider, blockholder and institucional ownership on firm-risk taking. Academy of Management Journal, v. 39, p. 441-463, 1996.

RaP Rio de Janeiro 41(1):49-62, Jan./Fev. 2007 